\title{
Point of care ultrasound training in Canadian emergency medicine residency programs
}

\author{
Daniel J. Kim ${ }^{1,2}(D) \cdot$ Paul Olszynski ${ }^{3} \cdot$ David J. W. Smith ${ }^{1} \cdot$ Elizabeth Lalande $^{4} \cdot$ Michael Y. Woo $^{5}$
}

Received: 26 August 2021 / Accepted: 17 January 2022 / Published online: 3 March 2022

(c) The Author(s), under exclusive licence to Canadian Association of Emergency Physicians (CAEP)/ Association Canadienne de Médecine d'Urgence (ACMU) 2022

\begin{abstract}
Objectives Point of care ultrasound (POCUS) has been endorsed as an important clinical tool by the Canadian Association of Emergency Physicians (CAEP) and is a training objective of emergency medicine (EM) residency programs accredited by both the Royal College of Physicians and Surgeons of Canada (RCPSC) and the College of Family Physicians of Canada (CFPC). Our objectives are to describe the national state of POCUS training in RCPSC-EM and CFPC-EM residency programs and to evaluate the implementation of the CAEP core POCUS curriculum in these programs.

Methods This was an online survey study of all POCUS education leads for both RCPSC-EM and CFPC-EM programs. The survey queried participants on program demographics, description of POCUS training, methods of POCUS assessment, and implementation of the CAEP core POCUS curriculum.

Results The response rate was 100\% (39/39). All RCPSC-EM and CFPC-EM programs provide POCUS training for their residents, and 100\% of RCPSC-EM programs and 91\% (20/22) of CFPC-EM programs have a POCUS lead. All programs provide POCUS training for their residents, but there is variability in how POCUS is introduced to residents, ongoing POCUS instruction provided throughout residency, and POCUS assessment. Only 47\% (8/17) of RCPSC-EM and 32\% (7/22) of CFPC-EM programs have a quality assurance process for the use of POCUS by their residents. POCUS leads believe their residents are proficient in the CAEP core POCUS applications by the end of training except for advanced cardiac and thoracic ultrasound.

Conclusions POCUS training in Canadian EM programs is prevalent, but there is variability in support for POCUS leads, delivery of training, determination of proficiency, and presence of quality assurance. While almost all programs deliver POCUS education aligning with the CAEP core POCUS curriculum position statement, more support is required both locally and nationally for sharing best practices for POCUS education.
\end{abstract}

Keywords Point of care ultrasound $\cdot$ POCUS $\cdot$ Emergency medicine $\cdot$ Education $\cdot$ Residency $\cdot$ Training $\cdot$ Assessment . Curriculum

Daniel J. Kim

dkim000@gmail.com

1 Department of Emergency Medicine, University of British Columbia, Vancouver, BC, Canada

2 Department of Emergency Medicine, Vancouver General Hospital, 855 12th Ave W, Vancouver, BC V5Z 1M9, Canada

3 Department of Emergency Medicine, College of Medicine, University of Saskatchewan, Saskatoon, SK, Canada

4 Department of Emergency Medicine, Centre Hospitalier de l'Université Laval (CHUL), CHU de Québec, Université Laval, Québec, QC, Canada

5 Department of Emergency Medicine, University of Ottawa and Ottawa Hospital Research Institute, Ottawa, ON, Canada 


\section{Résumé}

Objectifs L'échographie ciblée a été reconnue comme un outil clinique important par l'Association canadienne des médecins d'urgence (ACMU) et constitue un objectif de formation des programmes de résidence en médecine d'urgence (MU) agréés par le Collège royal des médecins et chirurgiens du Canada (CRMCC) et le Collège des médecins de famille du Canada (CMFC). Nos objectifs sont de décrire l'état national de la formation en échographie ciblée dans les programmes de résidence du CRMCC(MU) et du CMFC(MU) et d'évaluer la mise en œuvre du programme d'études en échographie ciblée de base d'ACMU dans ces programmes.

Méthodes Il s'agissait d'une étude par sondage en ligne auprès de tous les responsables de l'éducation en échographie ciblée pour les programmes CRMCC(MU) et CMFC(MU). L'enquête interrogeait les participants sur les données démographiques du programme, la description de la formation en échographie ciblée, les méthodes d'évaluation en échographie ciblée et la mise en œuvre du programme de base en échographie ciblée d'ACMU.

Résultats Le taux de réponse a été de 100\% (39/39). Tous les programmes CRMCC(MU) et CMFC(MU) offrent une formation en échographie ciblée à leurs résidents, et $100 \%$ des programmes CRMCC(MU) et $91 \%$ (20/22) des programmes CMFC(MU) ont un responsable d'échographie ciblée. Tous les programmes offrent une formation en échographie ciblée à leurs résidents, mais la façon dont l'échographie ciblée est présentée aux résidents, l'enseignement continu d'échographie ciblée tout au long de la résidence et l'évaluation de l'échographie ciblée varient. Seulement $47 \%$ (8/17) des programmes $\mathrm{CRMCC}(\mathrm{MU})$ et $32 \%$ (7/22) des programmes $\mathrm{CMFC}(\mathrm{MU})$ ont un processus d'assurance de la qualité pour l'utilisation d'une échographie ciblée par leurs résidents. Les responsables d'échographie ciblée estiment que leurs résidents maîtrisent les applications de base en échographie ciblée d'ACMU à la fin de la formation, sauf pour l'échographie cardiaque et thoracique avancée.

Conclusions La formation en échographie ciblée dans les programmes canadiens de MU est répandue, mais il existe une variabilité dans le soutien aux responsables d'échographie ciblée, la prestation de la formation, la détermination de la compétence et la présence d'une assurance qualité. Bien que presque tous les programmes offrent une formation en échographie ciblée qui s'harmonise avec l'énoncé de position du curriculum de base d'ACMU, il faut davantage de soutien à l'échelle locale et nationale pour partager les pratiques exemplaires en matière d'éducation en échographie ciblée.

\section{Clinician's capsule}

What is known about the topic?

POCUS is a core competency or training objective of both RCPSC emergency medicine and CFPC-EM residency programs.

\section{What did this study ask?}

What is the current state of POCUS training in Canadian emergency medicine residency programs?

\section{What did this study find?}

POCUS training is ubiquitous, but there is variability in delivery of training, determination of proficiency, and presence of quality assurance.

Why does this study matter to clinicians? Standardization of POCUS training will align POCUS alongside other key emergency medicine skills recognized within the completion of an emergency medicine residency.

\section{Introduction}

Point of care ultrasound (POCUS) is the real time use of ultrasound at the bedside, performed by the clinical provider, to either answer a focused clinical question or to improve procedural safety [1]. It has been endorsed as an important clinical skill by the Canadian Association of Emergency Physicians (CAEP) [2-4], a core competency in the Royal College of Physicians and Surgeons of Canada (RCPSC) emergency medicine (EM) program in 2008 [5], and a terminal training objective in the College of Family Physicians of Canada (CFPC) EM program in 2010 [6].

In 2015, the CAEP Emergency Ultrasound Committee formed the Emergency Ultrasound Curriculum Working Group. This group used a modified Delphi method to determine a consensus for a national EM POCUS curriculum. The core applications included: focused assessment with sonography in trauma, identification of abdominal aortic aneurysm, identification of intrauterine pregnancy by transabdominal approach, thoracic ultrasound, focused cardiac ultrasound, and ultrasound-guided vascular access [7]. These core POCUS applications were formally endorsed by CAEP in a position statement in 2018 [8].

It has been almost a decade since EM POCUS training was characterized in detail in Canada [9]. Over that time, 
there has been tremendous change in the POCUS landscape, including the transition of RCPSC-EM programs to competency-based medical education [10], improved access to machines and handhelds, and high-quality open access POCUS educational resources. Our objectives are to describe the national state of POCUS training in RCPSC-EM and CFPC-EM residency programs and to evaluate the implementation of the CAEP core POCUS curriculum in these programs.

\section{Methods}

\section{Study design}

This was an online survey study approved by the University of British Columbia Behavioural Research Ethics Board (No. H21-00282).

\section{Study setting and population}

All POCUS education leads in Canada for both RCPSCEM and CFPC-EM programs were invited to participate. Online Resource Appendix A details the identification of these individuals.

\section{Study protocol}

The survey instrument (Online Resource Appendix B) was designed by the study investigators based on previously published survey studies of POCUS training [9, 11, 12]. Online Resource Appendix A details the development and delivery (in March 2021) of the survey.

\section{Data analysis}

Data were analyzed with Microsoft Excel (Microsoft Corp., Redmond, WA), and descriptive statistics are reported using medians, interquartile ranges (IQRs), and ranges or number and proportion where appropriate.

\section{Results}

All 39 Canadian EM POCUS education leads completed the survey for a response rate of $100 \%$ (Online Resource Appendix C). All RCPSC-EM and CFPC-EM programs provide POCUS training for their residents. RCPSC-EM programs have a median of 22 residents $(\mathrm{IQR}=15-30)$ and CFPC-EM programs have a median of 7 residents (IQR = 6-9). All RCPSC-EM programs have a POCUS lead for their residency program or primary hospital, and 91\% (20/22) of CFPC-EM programs have a POCUS lead. Online Resource Appendix D breaks down the source of funding for this position.

$65 \%$ (11/17) of RCPSC-EM programs have an affiliated POCUS fellowship, introduced a median of 10 years ago, and 45\% (10/22) of CFPC-EM programs have an affiliated POCUS fellowship, which started a median of 4 years ago.

All programs provide POCUS training for their residents. Table 1 describes both diagnostic POCUS applications and ultrasound-guided procedures taught to residents. Online Resource Appendix E describes how POCUS is introduced to residents, ongoing POCUS instruction provided throughout residency, and POCUS instructional material provided to residents by their program. Online Resource Appendix $\mathrm{F}$ breaks down the specialties providing POCUS training to residents.

Online Resource Appendix G describes program's methods of determining proficiency in POCUS. The majority of programs had requirements for number of POCUS scans to be completed by the end of training [71\% (12/17) in RCPSCEM programs and 73\% (16/22) in CFPC-EM programs]. Less than half of programs have a departmental quality assurance process overseeing use of POCUS by their residents [47\% (8/17) of RCPSC-EM programs and 32\% (7/22) of CFPC-EM programs].

Online Resource Appendix $\mathrm{H}$ outlines the integration of POCUS training and assessment in RCPSC-EM competency-based medical education.

Online Resource Appendix I reports familiarity with and implementation of the CAEP core POCUS curriculum.

\section{Discussion}

\section{Interpretation of findings}

Our data demonstrate that all Canadian EM programs provide POCUS training for their residents. Over half of programs have an affiliated POCUS fellowship. There is considerable variability from site to site in the way POCUS training is delivered (Online Resource Appendix E). The most common method of determining POCUS proficiency in RCPSC-EM programs is by competency committee determination based on entrustable professional activity (EPA) achievement (76\%), whereas the most common method of determining proficiency in CFPC-EM programs is by certification from an external organization (73\%). This is surprising given the 2019 CAEP POCUS position statement recommends that completion of residency should provide evidence of competency in POCUS [4]. External commercial courses and external certifications seem to be favored by a majority 
Table 1 POCUS applications and ultrasound-guided procedures taught to emergency medicine residents in Canada for RCPSC and CFPC emergency medicine programs

\begin{tabular}{|c|c|c|c|c|}
\hline \multirow{2}{*}{ POCUS applications } & \multicolumn{2}{|c|}{$\begin{array}{l}\text { RCPSC- } \\
\text { EM } \\
\text { programs } \\
N=17 \\
\text { No. }(\%)\end{array}$} & \multicolumn{2}{|c|}{$\begin{array}{l}\text { CFPC- } \\
\text { EM pro- } \\
\text { grams } \\
N=22 \\
\text { No. }(\%)\end{array}$} \\
\hline & & & & \\
\hline None & 0 & $(0)$ & 0 & $(0)$ \\
\hline Focused Assessment with Sonography for Trauma (FAST) & 17 & $(100)$ & 22 & $(100)$ \\
\hline Extended-FAST (E-FAST for pneumothorax and pleural effusion/hemothorax) & 17 & $(100)$ & 22 & $(100)$ \\
\hline Pelvic/first trimester intrauterine pregnancy (IUP) via transabdominal approach & 17 & $(100)$ & 22 & $(100)$ \\
\hline Pelvic/first trimester IUP via transvaginal approach & 11 & $(65)$ & 18 & $(82)$ \\
\hline Aorta for abdominal aortic aneurysm (AAA) & 17 & $(100)$ & 22 & $(100)$ \\
\hline Basic cardiac (subxiphoid for pericardial effusion and cardiac standstill) & 17 & $(100)$ & 22 & $(100)$ \\
\hline $\begin{array}{l}\text { Advanced cardiac (parasternal long axis, parasternal short axis, and apical } 4 \\
\text { chamber for gross assessment of left ventricular and right ventricular func- } \\
\text { tion) }\end{array}$ & 17 & $(100)$ & 19 & $(86)$ \\
\hline Thoracic for pulmonary edema and consolidation & 17 & $(100)$ & 19 & $(86)$ \\
\hline Biliary & 13 & (76) & 14 & $(64)$ \\
\hline Renal/urinary & 13 & (76) & 12 & $(55)$ \\
\hline Deep vein thrombosis (DVT) & 11 & $(65)$ & 10 & $(45)$ \\
\hline Soft tissue/musculoskeletal & 12 & $(71)$ & 10 & $(45)$ \\
\hline Ocular & 10 & (59) & 6 & $(27)$ \\
\hline \multicolumn{5}{|l|}{ Ultrasound-guided procedures } \\
\hline None & 0 & $(0)$ & 2 & (9) \\
\hline Arterial line placement & 10 & (59) & 6 & $(27)$ \\
\hline Arthrocentesis & 13 & (76) & 10 & $(45)$ \\
\hline Central venous catheter insertion & 17 & $(100)$ & 19 & $(86)$ \\
\hline Cutaneous abscess incision and drainage & 11 & $(65)$ & 9 & $(41)$ \\
\hline F oreign body removal & 7 & $(41)$ & 7 & $(32)$ \\
\hline Lumbar puncture & 8 & $(47)$ & 6 & $(27)$ \\
\hline Nerve blocks & 13 & (76) & 11 & $(50)$ \\
\hline Paracentesis & 13 & (76) & 10 & $(45)$ \\
\hline Pericardiocentesis & 8 & $(47)$ & 7 & $(32)$ \\
\hline Peripheral venous catheter insertion & 14 & $(82)$ & 14 & (64) \\
\hline Peritonsillar abscess incision and drainage & 4 & (24) & 2 & (9) \\
\hline Thoracentesis & 12 & $(71)$ & 8 & (36) \\
\hline Transvenous pacemaker insertion & 3 & $(18)$ & 3 & (14) \\
\hline
\end{tabular}

POCUS point of care ultrasound, RCPSC Royal College of Physicians and Surgeons of Canada, CFPC College of Family Physicians of Canada, EM emergency medicine of CFPC-EM programs. This may partially be explained by the challenges of delivering EM training in a focused 1 year program, as well as limited faculty expertise. The variation in training, educational resources, and determination of proficiency suggests the POCUS education of Canadian EM residents is often regional and site specific with differences between RCPSC-EM and CFPC-EM programs.

Almost all POCUS leads are familiar with the CAEP POCUS curriculum and core applications [7, 8]. Although only $22 \%$ of respondents reported that it changed the way they delivered POCUS education, almost all programs teach all the core applications. POCUS leads feel their residents may not develop proficiency in advanced cardiac and thoracic ultrasound for pulmonary edema and consolidation by the end of training. This concern was more common in CFPC-EM programs compared to RCPSCEM programs. Despite the seemingly limited effect of the CAEP core POCUS curriculum statement, one respondent comments: "There was a lot of resistance from [the] local EM program director to expand [our] POCUS program. The CJEM POCUS [position statement] article helped [bring] acceptance to ... expand training." 


\section{Comparison to previous studies}

In 2011, $12 \%$ of CFPC-EM programs did not include POCUS as part of the formal residency curriculum [9]. Since that time, there has been integration of POCUS training into all Canadian EM programs. There has also been dramatic growth in POCUS fellowships, with more than half of programs having an affiliated fellowship; in 2011, only $17 \%$ of EM programs had an affiliated fellowship [9]. This may partially be explained by the approval of an Area of Focused Competence Diploma for acute care POCUS in 2016 by the RCPSC [13], with the first program receiving accreditation in 2020. Programs with a fellowship have a positive impact on the POCUS educational experience of their residents, as EM residents perform more scans and use more advanced applications in programs with a POCUS fellowship [14].

Less than half of programs have a departmental quality assurance process overseeing use of POCUS by their residents: $47 \%$ in RCPSC-EM programs and 32\% in CFPC-EM programs. There has been minimal movement in this area of POCUS administration; in 2011, only $31 \%$ of RCPSC-EM and $47 \%$ of CFPC-EM programs had a quality assurance process for the use of POCUS by EM staff and residents [9].

\section{Strengths and limitations}

The core strength of this study is the response rate of $100 \%$. It presents the most complete picture of POCUS residency training in Canada at this time. However, the responses are based on the perceptions of POCUS leads (or program directors in the absence of a dedicated POCUS lead), and as such, they may not fully reflect the positions of the actual programs or the POCUS training experience of the programs' residents. It is also possible that residency program directors may not be as familiar with their program's POCUS training as a dedicated POCUS lead might be. Additionally, there are inherent limitations with online survey studies, including a possibility of misinterpretation of questions or answer choices by respondents.

\section{Clinical implications}

Almost all programs have a dedicated POCUS lead, but of concern is that $27 \%(10 / 37)$ of these positions are not funded. The 2019 CAEP POCUS position statement highlights the need for the appropriate support of POCUS lead positions, especially considering the physician time required to run a POCUS program that includes quality assurance and improvement, staff credentialing, machine purchase and maintenance, and delivery of POCUS education [4]. POCUS quality assurance is best practice [4] and is a specific area of POCUS administration needing improvement. Implementation of a robust POCUS quality assurance process is challenging, requiring capital investment by hospital leadership for the purchase and information technology support to allow for archiving of clips from the department's ultrasound machines [15]. To implement POCUS quality assurance programs more broadly, there needs to be more financial support from the local department and hospital level, and the regional health system and province level.

Our study results should reassure local POCUS directors and emergency department heads that POCUS training and proficiency assessment in Canada are increasingly robust. Completion of EM residency from most programs confers competency in POCUS.

\section{Conclusion}

POCUS training in Canadian EM programs is prevalent, and almost all programs have a dedicated POCUS lead overseeing their resident POCUS training. However, there is variability in support for POCUS leads, delivery of training, determination of proficiency, and presence of quality assurance programs. Although the CAEP POCUS curriculum position statement has been helpful with introducing consistency in the core POCUS applications that are taught, more support is required both locally and nationally for quality assurance programs and sharing best practices with POCUS education that can be adapted to each program's unique setting.

Supplementary Information The online version contains supplementary material available at https://doi.org/10.1007/s43678-022-00269-1.

\section{Declarations}

Conflict of interest PO, DJWS, EL, and MYW do not report any conflicts of interest. DJK is on the medical advisory board of Clarius Mobile Health.

\section{References}

1. Moore CL, Copel JA. Point-of-care ultrasonography. N Engl J Med. 2011;364(8):749-57.

2. Socransky S. Emergency department targeted ultrasound: 2006 update. CJEM. 2006;8(3):170-4.

3. Henneberry R, Hanson A, Healey A, Hebert G, Ip U, Mensour $\mathrm{M}$, et al. Use of point of care sonography by emergency physicians. CJEM. 2012;14(2):106-12.

4. Lewis D, Rang L, Kim D, Robichaud L, Kwan C, Pham C, et al. Recommendations for the use of point-of-care ultrasound (POCUS) by emergency physicians in Canada. CJEM. 2019;21(6):721-6.

5. Royal College of Physicians and Surgeons of Canada. Objectives of Training in the Specialty of Emergency Medicine. Available at: https://www.royalcollege.ca/rcsite/documents/ ibd/emergency_otr_e.pdf. Accessed 6 Jan 2021. 
6. The College of Family Physicians of Canada. Emergency Medicine Key Features of the Priority Topics for the Assessment of Competence in Family Medicine at the Enhanced Skills Level. Available at: https://www.cfpc.ca/CFPC/media/Images/PDF/ EM_KF-2017_Final_ENG.pdf. Accessed 6 Jan 2021.

7. Olszynski P, Kim D, Chenkin J, Rang L. The core emergency ultrasound curriculum project: a report from the curriculum working group of the CAEP emergency ultrasound committee. CJEM. 2018;20(2):176-82.

8. Olszynski P, Kim DJ, Chenkin J, Rang L, Group members of the CEUC curriculum working. The CAEP emergency ultrasound curriculum-objectives and recommendations for implementation in postgraduate training: executive summary. CJEM. 2018;20(5):736-8.

9. Kim DJ, Theoret J, Liao MM, Hopkins E, Woolfrey K, Kendall $\mathrm{JL}$. The current state of ultrasound training in Canadian emergency medicine programs: perspectives from program directors. Acad Emerg Med. 2012;19(9):E1073-1078.

10. Royal College of Physicians and Surgeons of Canada. CBD Launch Schedule. Available at: https://www.royalcollege.ca/ rcsite/documents/cbd-community-touchpoint/cbd-rollout-sched ule-e. Accessed 6 Jan 2021.

11. Mok D, Schwarz SKW, Rondi K. Point-of-care ultrasonography in Canadian anesthesiology residency programs: a national survey of program directors. Can J Anaesth. 2017;64(10):1023-36.

12. Mosier JM, Malo J, Stolz LA, Bloom JW, Reyes NA, Snyder LS, et al. Critical care ultrasound training: a survey of US fellowship directors. J Crit Care. 2014;29(4):645-9.

13. Royal College of Physicians and Surgeons of Canada. Acute Care Point of Care Ultrasonography (POCUS). Available at: https:// www.royalcollege.ca/rcsite/documents/ibd/acute-care-ultrasonog raphy-pocus-info-e. Accessed 18 Aug 2021.

14. Adhikari S, Raio C, Morrison D, Tsung J, Leech S, Meer J, et al. Do emergency ultrasound fellowship programs impact emergency medicine residents' ultrasound education? J Ultrasound Med. 2014;33(6):999-1004.

15. Kim DJ, Thiessen M, Strony R. Point-of-care ultrasound credentialing: big picture principles for big health systems. Jt Comm J Qual Patient Saf. 2020;46(8):435-7. 